\title{
CORR $^{\circledR}$ ORS Richard A. Brand Award: Clinical Trials of a New Treatment Method for Adhesive Capsulitis
}

\author{
Marie A. Badalamente, Edward D. Wang
}

Received: 24 November 2015/Accepted: 22 April 2016/Published online: 9 May 2016

(C) The Association of Bone and Joint Surgeons (B) 2016

\begin{abstract}
Background Conservative and even surgical management of adhesive capsulitis often is prolonged and painful. Management of adhesive capsulitis is lacking evidencebased controlled clinical trials.

Questions/Purposes We asked: (1) Does a collagenase clostridium histolyticum $(\mathrm{CCH})$ injection lyse shoulder capsule collagen in adhesive capsulitis and at what dose?

(2) Can a shoulder capsule injection be administered extraarticularly? (3) Do CCH injections result in better scores for pain and function than can be achieved with physical therapy among patients with adhesive capsulitis?
\end{abstract}

The Stony Brook University Research Foundation and MAB and EDW received funding from Auxilium Pharmaceutical, Inc.

One of the authors certifies that she (MAB), or a member of her immediate family, has or may receive payments or benefits, during the study period, an amount of USD less than USD 10,000, from Auxilium Pharmaceutical, Inc (Malvern, PA, USA).

One of the authors certifies that he (EDW), or a member of his immediate family, has or may receive payments or benefits, during the study period, an amount of USD less than USD 10,000, from Auxilium Pharmaceutical, Inc (Malvern, PA, USA).

All ICMJE Conflict of Interest Forms for authors and Clinical Orthopaedics and Related Research ${ }^{\circledR}$ editors and board members are on file with the publication and can be viewed on request.

Clinical Orthopaedics and Related Research ${ }^{\mathbb{R}}$ neither advocates nor endorses the use of any treatment, drug, or device. Readers are encouraged to always seek additional information, including FDAapproval status, of any drug or device prior to clinical use.

Each author certifies that his or her institution approved the human protocol for this investigation, that all investigations were conducted in conformity with ethical principles of research, and that informed consent for participation in the study was obtained.

M. A. Badalamente ( $)$, E. D. Wang

Department of Orthopaedics, Stony Brook University Medical

Center, Stony Brook, NY 11794, USA

e-mail: Marie.Badalamente@stonybrookmedicine.edu
Methods First, 60 patients with adhesive capsulitis were evaluated by clinical examination. To make the diagnosis of adhesive capsulitis, a patient had to have restricted active ROM of at least $60^{\circ}$ in total active ROM in the affected shoulder compared with the unaffected contralateral shoulder; with the scapula stabilized, external rotation with the elbow at the side was a very important determinant. Patients were randomized to receive a single injection of $0.5 \mathrm{~mL}$ placebo or $0.145,0.29$, or $0.58 \mathrm{mg} \mathrm{CCH}$. All 60 patients were followed up at 30 days. After that, if patients did not attain treatment thresholds they were eligible for up to five open-label 0.58 -mg collagenase injections. For the longer-term followup in the open-label phase, 53 patients (83\%) were followed to 12 months, 46 (77\%) for 24 months, 36 (60\%) for 36 months, 37 (62\%) for 48 months, and $25(42 \%)$ for 60 months. The extraarticular injection was directed at the anterior shoulder capsule with the patient in the supine position. To prove that these injections could be delivered reliably to the anterior shoulder capsule extraarticularly, the next study involved volunteers without adhesive capsulitis, in which 10 volunteers received a 10$\mathrm{mL}$ injection of normal saline under ultrasound guidance. Finally, to determine the efficacy and dosing of $\mathrm{CCH}$, four cohorts of 10 patients received up to three ultrasoundguided injections separated by 21 days. These injections were administered at one of four dose-volume levels. A fifth cohort of 10 patients was used as a control group and performed standardized home shoulder exercises only. All patients performed standardized home shoulder exercises three times daily. For Study 3, followup was at 22, 43, 64, and 92 days. No patients were lost to followup.

Results In the first study, a single $\mathrm{CCH}$ injection did not provide clinically important improvements from baseline in active ROM, passive ROM, and function and pain scores compared with patients who received placebo. Ultrasound 
guidance confirmed extraarticular injection of the shoulder capsule in Study 2. The $\mathrm{CCH}$ injection was more effective than exercise therapy alone at $0.58 \mathrm{mg} / 1 \mathrm{~mL}$ and $0.58 \mathrm{mg} / 2$ $\mathrm{mL}$ compared with exercise only in the primary measure of efficacy (active forward flexion) as shown in Study 3. For active forward flexion the mean in degrees in the $0.58 \mathrm{mg} / 2$ $\mathrm{mL}$ group was $38^{\circ}$ compared with $12^{\circ}$ in the exercise-only group $(\mathrm{p}=0.03)$. For active forward flexion the mean in the $0.58 \mathrm{mg} / 1 \mathrm{~mL}$ group was $43^{\circ}$ compared with $12^{\circ}$ in the exercise-only group $(\mathrm{p}=0.01)$.

Conclusions Extraarticular injections of $\mathrm{CCH}$ for treatment of adhesive capsulitis were well tolerated and seem effective compared with exercise therapy. Future FDAregulated clinical trials must verify $\mathrm{CCH}$ injection therapy for adhesive capsulitis.

Level of Evidence Level II, therapeutic study.

\section{Introduction}

Adhesive capsulitis, commonly known as frozen shoulder, is characterized by a painful, gradual loss of active and passive glenohumeral motion resulting from fibrosis and ultimate contracture of the glenohumeral joint capsule [8, 9]. Adhesive capsulitis affects $2 \%$ to $5 \%$ of the adult population, predominantly women in the 40- to 60-year-old range $[8,9]$. The condition is progressive, with three recognized stages. In Stage 1, the "freezing" stage, pain begins and patients lose ROM. Freezing typically lasts from 6 weeks to 9 months. In Stage 2, the "frozen" stage, painful symptoms may improve, but the stiffness remains. During the 4 to 6 months of the "frozen" stage, daily activities may be very difficult. In Stage 3, the "thawing" stage, shoulder motion slowly improves. Complete return to normal or close to normal strength and motion typically takes from 6 months to 2 years [8,9].

Management of adhesive capsulitis is directed at the relief of pain and restoration of motion and function. Physical therapy and pharmacologic measures, such as intraarticular steroid injections, and more invasive measures, such as manipulation under anesthesia or arthroscopic capsular release, are used to treat adhesive capsulitis [8, 9]. However, in the absence of prospective, well-controlled, randomized clinical studies, controversies remain regarding which treatment strategies to use $[2,6,8$, 11].

Collagenase clostridium histolyticum $(\mathrm{CCH})$ consists of two synergistic collagenases (CCH-I and CCH-II). It is an FDA-approved enzymatic injection treatment for Dupuytren's contracture in adult patients with a palpable cord [12]. CCH injection treatment for Dupuytren's contracture was first developed at Stony Brook University Medical
Center (Stony Brook, NY, USA) [5]. Considering that the $\mathrm{CCH}$ mechanism of action on collagen might be the same for adhesive capsulitis as it is in Dupuytren's contracture, we thought that the effects of $\mathrm{CCH}$ might prove clinically beneficial to patients with adhesive capsulitis.

The objectives of the three clinical trials performed were first to define a minimum safe and effective dose of $\mathrm{CCH}$ for injection in patients with adhesive capsulitis compared with placebo controls. Next, we sought to confirm the shoulder injection method using ultrasound guidance. Finally, we again sought to test for a minimum safe and effective $\mathrm{CCH}$ dose and volume of injections in patients with adhesive capsulitis.

Specifically, we asked: (1) Does a CCH injection lyse shoulder capsule collagen in adhesive capsulitis and at what dose? (2) Can a shoulder capsule injection be administered extraarticularly? (3) Do $\mathrm{CCH}$ injections result in better scores for pain and function than can be achieved with physical therapy among patients with adhesive capsulitis?

\section{Methods}

\section{Study Design and Setting}

Study 1 was randomized, blinded, and placebo controlled. Study 2 was performed using volunteers without adhesive capsulitis and all received saline injections. Study 3 was randomized but not blinded to $\mathrm{CCH}$ dose groups versus exercise-only control patients.

\section{Participants}

\section{Study 1. One CCH Injection}

Sixty patients (47 women and 13 men) were enrolled at one site (Stony Brook Medical Center). Their mean age was 52 years. Screening included medical history, physical examination, and clinical examination of both shoulders, including ROM measurements of both shoulders. Radiographs were performed. Patients with other shoulder disorders were excluded. Mean disease duration was 17 months.

\section{Study 2. Volunteers Without Adhesive Capsulitis}

Ten adult volunteers were enrolled. Only individuals judged to be in good health and with no current or past shoulder disorders were enrolled. There were eight women 
and two men, with a mean age of 45 years (range, 38-52 years).

\section{Study 3. CCH Dose Range-volume versus Exercise-only Controls)}

Eligible adult patients were those with 3 to 12 months of unilateral idiopathic adhesive capsulitis (Stage 2, frozen stage) of one shoulder, with normal ROM in the contralateral shoulder. Other eligibility criteria applied. Fifty patients (40 women, 10 men) with a mean age of $54 \pm 8$ years were enrolled at 11 sites in the US.

Men and women were eligible if they (1) were 18 years or older; (2) were surgically sterile or not pregnant (if a woman). Contraceptive measures had to be taken by nonsurgically sterile women for at least one menstrual cycle before enrollment and after the end of the study. Men and women also were eligible if they (3) had unilateral idiopathic adhesive capsulitis of one shoulder for 3 to 12 months and be in Stage 2 (frozen stage); all other shoulder disorders were excluded based on MRI; and clinical examination of restricted active and passive ROM, especially internal and external rotation, helped to confirm the diagnosis of adhesive capsulitis. Finally, men and women also were eligible if they (4) had restricted active ROM in the affected shoulder, specifically, compared with the contralateral shoulder, a deficit of at least $60^{\circ}$ in total active $\mathrm{ROM}$ in the affected shoulder, or a deficit of at least $30^{\circ}$ in active ROM in one or more of forward flexion, abduction, or external rotation, or internal rotation with the elbow up to $90^{\circ}$ abduction.

Patients were ineligible if they (1) were men who intended to father a child during the study or who had pregnant partners; (2) were women who were, or who intended to become, pregnant during the study or who were breastfeeding; (3) had received prior treatment for adhesive capsulitis or were planning to receive treatment for adhesive capsulitis at any time during the study including but not limited to physical therapy or acupuncture within 2 weeks before the first injection of $\mathrm{CCH}$, intraarticular or intrabursal injection(s) of lidocaine, suprascapular nerve blocks, corticosteroids, electroanalgesic, and/or thermoanalgesic modalities within 1 month before the screening visit, intraarticular or intrabursal injection(s) of sodium hyaluronate within 3 months before the screening visit, or glenohumeral distension arthrography and/or surgical intervention (including shoulder manipulation under anesthesia) at any time; (4) had any of the following conditions in the affected shoulder: adhesive capsulitis owing to traumatic injury, pain at rest that was 4 or greater on an 11-point pain scale, active subacromial impingement, calcified tendinitis, glenohumeral joint arthritis, arthrosis, chondrolysis, subscapularis tendon rupture, or other rotator cuff injuries; (5) had uncontrolled hypertension, diabetes, or thyroid disease, a history of thrombosis or postthrombosis syndrome, physical impairment that precluded performing the protocol-defined exercises, active infection in the area to be treated, clinically significant neurologic disease, coagulation disorder or use of an anticoagulant (except for $\leq 150 \mathrm{mg}$ aspirin) within 7 days before injection of $\mathrm{CCH}$, known active hepatitis $\mathrm{B}$ or $\mathrm{C}$, or any other significant medical condition that, in the investigator's opinion, precluded participation in the trial; (6) had

Table 1. Successful treatment thresholds

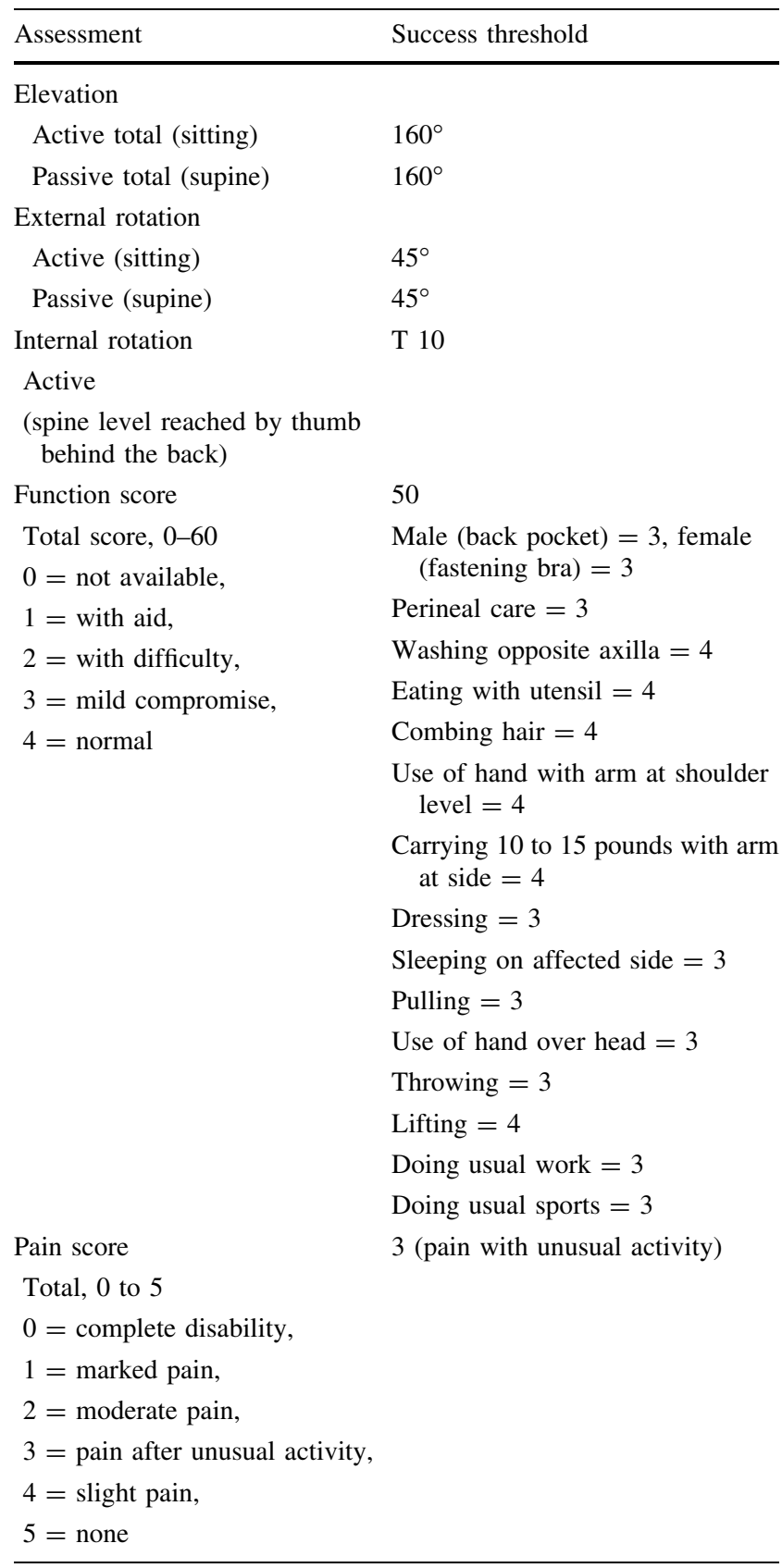


received an investigational drug or treatment within 30 days before the first dose of $\mathrm{CCH}$, had received collagenase for the treatment of adhesive capsulitis or were planning to be treated with commercial $\mathrm{CCH}$ at any time during the study, had a known systemic allergy to collagenase or any other excipient of the $\mathrm{CCH}$ formulation, or to any other procedural medication, or were unable to undergo radiographs or MRI of the affected shoulder.

\section{Description of Experiment, Treatment, or Surgery}

\section{Study 1. One CCH Injection}

Patients were randomized to receive a single injection of placebo (buffer) or $0.145 \mathrm{mg}, 0.29 \mathrm{mg}$, or $0.58 \mathrm{mg}$ of CCH (Xiaflex ${ }^{\circledR}$; Auxilium Pharmaceuticals, Inc, Malvern, PA, USA) dissolved in sterile buffer $(0.9 \%$ saline and $2 \mathrm{mmol} / \mathrm{L}$ $\mathrm{Ca}+{ }^{2}$ ). The volume for all injections was $0.5 \mathrm{~mL}$. The investigators were blinded to treatment and treatment blinding was broken 1 month after injection of the 60th patient enrolled.

Patients were positioned supine, and the affected arm was passively externally rotated with the elbow at the side to the patient's tolerance of pain. Landmarks were identified by palpating the bicipital groove and the coracoid process, and the injection site was midway in the coronal plane between these two landmarks. After washing the area with Betadine ${ }^{\circledR}$ (Purdue Pharma LP, Stamford, CT, USA) and ethyl alcohol, local anesthesia (10 $\mathrm{mL}$ of $1 \%$ lidocaine solution) was injected extraarticularly with a 20-gauge needle. Next, the $\mathrm{CCH}$ injection was administered in the same location using the same needle track and a blunttipped Sprotte ${ }^{\circledR} 22$-gauge spinal needle (Medline Industries Inc, Mundelein, IL, USA) which is not capable of penetrating the joint capsule or the subscapularis tendon because the tip of the needle is blunt.

Patients were serially evaluated at $1,2,4,7,14$, and 30 days after injection using the American Shoulder and Elbow Surgeons' (ASES) assessment [4]. Measures included active-elevation, external rotation, internal rotation (spine level reached by thumb behind the back), passive elevation, external rotation at the side, external rotation at $90^{\circ}$, and function and pain scores. The function score comprised 14 categories, which are individually scored from 0 to 4 ; a score of 60 represents being fully functional. The pain score uses a Likert scale of 0 to 5 . A successful treatment threshold was defined as improvement in motion, pain, and function at 30 days after injection.

Successful treatment thresholds for each assessment were established (Table 1). Patients who did not achieve successful treatment thresholds had the option of receiving up to four additional $0.58-\mathrm{mg} / 0.5-\mathrm{mL} \mathrm{CCH}$ injections with a 5- to 6-week interval between each injection in an openlabel extension phase. After their last injection, patients were followed at 2, 3, 6, 9, and 12 months and annually thereafter for 60 months for longer-term evaluation of treatment.

Patients were assessed for adverse events at all study visits

\section{Study 2. Volunteers Without Adhesive Capsulitis}

Before the injection, an ultrasound image from the posterior aspect of the target shoulder was obtained with the volunteers lying on their sides. For an anterior injection, the volunteers were supine, and the arm was passively externally rotated with the elbow at the side. The anterior area was washed with chlorhexadine solution. Landmarks were identified by palpating the bicipital groove and the coracoid process and marked with a sterile surgical marker. The injection site was midway in the coronal plane between these two landmarks (Fig. 1).

Anesthesia and injection, with $10 \mathrm{~mL}$ sterile saline, was as described For Study 1 using ultrasound guidance (Fig. 2). Anterior and posterior ultrasound images were recorded in a serial fashion and timed. Imaging of the saline injection was blinded to the surgeon (EDW) who performed all injections.

\section{Study 3. CCH Dose Range-volume versus Exercise-only Controls}

Screening included medical history, physical examination, and clinical examination of both shoulders, which included ROM measurements of both shoulders and radiographs and MRI of the affected shoulder. Function and pain scores were performed using the ASES assessment [7]. Clinical laboratory assessments, vital signs, and 12-lead ECG also were included. Fifty patients were enrolled and randomized. All enrolled patients completed the study through followup on Day 92. All patients in Cohorts 1 through 4 received one dose of $\mathrm{CCH}$. Twenty-nine of 40 patients $(72.5 \%)$ received three $\mathrm{CCH}$ injections, five received two injections, and six received one injection. The mean duration of the current adhesive capsulitis event was 233 days. Self-reported compliance with the standardized home exercise regimen was $80 \%$ to $100 \%$.

Patients received injections of $\mathrm{CCH}$ plus standardized home exercise $(n=40)$ or home exercise only $(n=10)$. Cohort 1 received $0.29 \mathrm{mg} \mathrm{CCH} / 1.0 \mathrm{~mL}$; Cohort 2 received $0.58 \mathrm{mg} \mathrm{CCH} / 2.0 \mathrm{~mL}$; Cohort 3 received $0.58 \mathrm{mg} \mathrm{CCH} / 1.0$ $\mathrm{mL}$; and Cohort 4 received $0.58 \mathrm{mg} \mathrm{CCH} / 0.5 \mathrm{~mL}$. Up to three injections of $\mathrm{CCH}$, separated by at least 21 days, could 
Table 2. Pain and function assessment measures

\begin{tabular}{ll}
\hline Assessment & Description \\
\hline Pain & $\begin{array}{l}\text { 11-point VAS ranging from } 0 \text { (no pain at all) to } 10 \text { (pain as bad as it could be) in response to the question 'How bad is your pain } \\
\text { today?' }\end{array}$ \\
Function & $\begin{array}{l}\text { 4-point scale ( } 0 \text {, unable to do; } 1 \text {, very difficult; } 2 \text {, somewhat difficult; and 3, normal) describing the ability to perform certain tasks } \\
\text { (put on coat; sleep on the painful or affected side; wash back/hook bra in back; manage toileting; comb/wash hair; reach a high }\end{array}$ \\
& shelf; lift 10 pounds above shoulder; throw a ball overhand; do usual work [with list]; and do usual sport [with list])
\end{tabular}

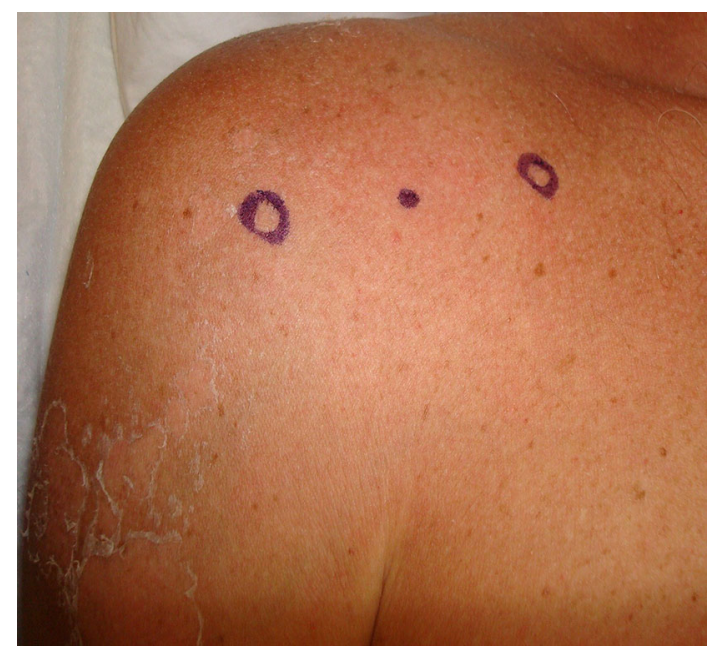

Fig. 1 Anatomic landmarkmarks of the anterior injection site midway between the tip of the coracoid and the bicipital groove are shown.

be given. The second and third injections were given if on the day of the scheduled injection, all retreatment criteria were met and no safety issues were identified. The retreatment criteria were active (sitting) and passive (supine) measurements of less than $140^{\circ}$ for forward flexion and abduction, and external rotation and internal rotation less than $30^{\circ}$ when measured with the humerus abducted as tolerated up to a maximum $90^{\circ}$. The second and third injections were not given if all "do not retreat" criteria were met. These "do no retreat" criteria were active and passive measurements of $160^{\circ}$ or greater for forward flexion and abduction, and external rotation and internal rotation of $45^{\circ}$ or greater with the humerus abducted as tolerated up to $90^{\circ}$. For patients who were between all retreatment or all do not retreat criteria, the second and third injections were given at the investigator's discretion.

All patients performed standardized home shoulder exercises (shoulder flexion with pulleys, sideways wall climbing for abduction, towel stretching for internal rotation, doorway external rotation stretching, and pendulum exercises for all planes) three times daily. Followups were at Days 22 ( \pm 7 days), 43 ( \pm 7 days), and 64 ( \pm 7 days).

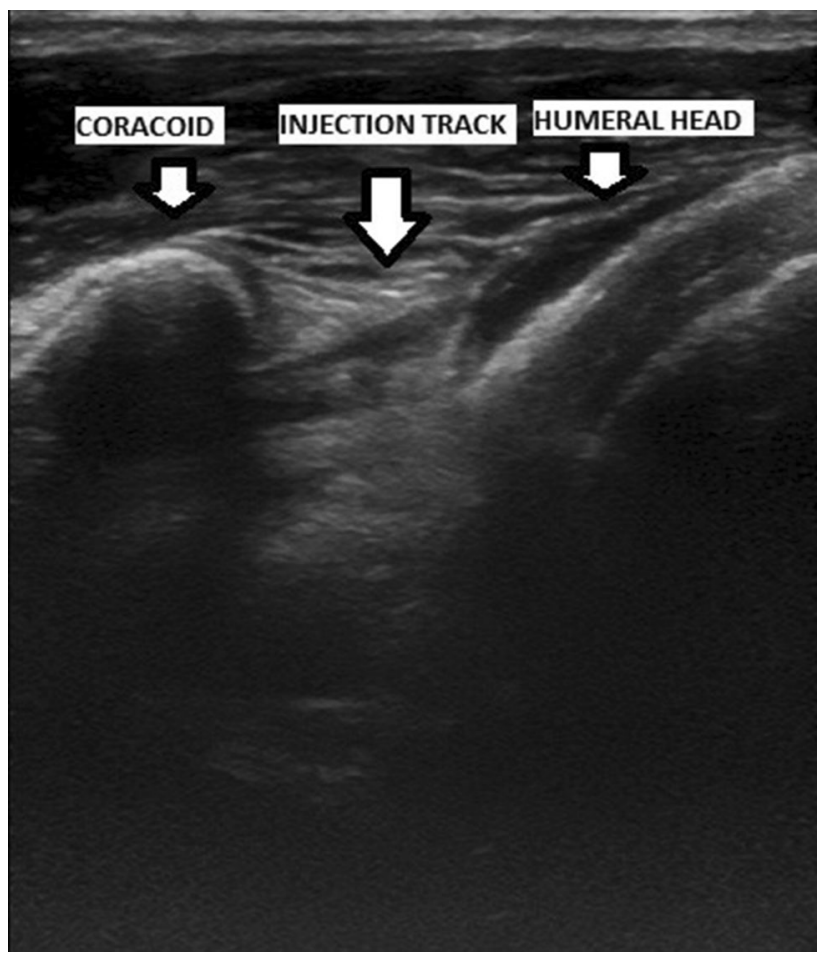

Fig. 2 An ultrasound image of the anatomic landmarks, including the humeral head and the injection track, is shown.

All patients in all cohorts returned for a final followup on Day 92 ( \pm 7 days).

Anesthesia and $\mathrm{CCH}$ injection were as described in Study 2 under ultrasound guidance.

Efficacy assessments were made by a blinded assessor (JP) and included active ROM (seated) and passive ROM (supine) for forward flexion, abduction, external rotation with the humerus up to $90^{\circ}$ abducted, and internal rotation with the humerus up to $90^{\circ}$ abducted. The primary efficacy endpoint was change from baseline, compared with Day 92, in active forward flexion of the affected shoulder. Active ROM, passive ROM, pain, and function (Table 2) were assessed in Cohorts 1 through 4 before each injection, 7 and 21 days after each injection, and on Day 92. In Cohort 5 the same assessments were performed on Days 1, 22, 43, 64, and 92. Pain and function scores in the affected shoulder 
were assessed using the ASES evaluation [7] (Table 2). The VAS pain score ranged from zero (no pain at all) to 10 (pain as bad as it can be). Each item on the functional scale was answered on a four-point scale of 0 (unable to do), 1 (very difficult), 2 (somewhat difficult), and 3 (normal).

Safety evaluations included clinical laboratory assessment and MRI of the affected shoulder in all five cohorts at baseline and on Day 92. At each visit, vital signs were measured and adverse events were assessed.

Variables, Outcome Measures, Data Sources, and Bias

\section{Study 1. One CCH Injection}

All 60 patients were followed up at 30 days. After that, if patients did not attain treatment thresholds they were eligible for up to five open-label 0.58 -mg collagenase injections. For the longer-term followup in the open-label phase, 53 patients $(83 \%)$ were followed to 12 months, 46 (77\%) for 24 months, 36 (60\%) for 36 months, 37 (62\%) for 48 months, and 25 (42\%) for 60 months.

The patients were serially evaluated at $1,2,4,7,14$, and 30 days after injection using the ASES assessment [4]. Measures included active elevation, external rotation, internal rotation (spine level reached by thumb behind the back), passive elevation, external rotation at the side, external rotation at $90^{\circ}$, and function and pain scores. The function score comprised 14 categories which are individually scored from 0 to 4 ; a score of 60 represents being fully functional. The pain score uses a Likert scale of 0 to 5. A successful treatment threshold was defined as improvement in motion, pain, and function at 30 days postinjection.

Successful treatment thresholds for each assessment were established (Table 1). Patients who did not reach successful treatment thresholds had the option of receiving up to four additional $0.58-\mathrm{mg} / 0.5-\mathrm{mL} \mathrm{CCH}$ injections with a 5- to 6-week interval between each injection in an openlabel extension phase. After the last injection, patients were followed at 2, 3, 6, 9, and 12 months and annually thereafter. For the longer-term followup in the open-label phase, 53 patients were followed to 12 months, 46 for 24 months, 36 for 36 months, 37 for 48 months, and 25 for 60 months. Patients were assessed for adverse events at all study visits.

\section{Study 2. Volunteers Without Adhesive Capsulitis}

Under direct observation using ultrasound imaging, after $1 \%$ lidocaine injection between the coracoid tip and bicipital groove, the Sprotte ${ }^{\circledR}$ needle was placed in the same needle track. Ten $\mathrm{mL}$ of sterile normal saline then was injected and the bolus of saline observed and timed until it disappeared.

\section{Study 3. CCH Dose Range-volume versus Exercise-only Controls}

Efficacy assessments included active ROM (seated) and passive ROM (supine) for forward flexion, abduction, external rotation with the humerus up to $90^{\circ}$ abducted, and internal rotation with the humerus up to $90^{\circ}$ abducted. The primary efficacy endpoint was change from baseline compared with Day 92 in active forward flexion of the affected shoulder. Active ROM, passive ROM, pain, and function (Table 2) were assessed by a blinded assessor (JP) in Cohorts 1 through 4 before each injection, 7 and 21 days after each injection, and on Day 92. In Cohort 5 the same assessments were performed by the blinded assessor (JP) on Days 1, 22, 43, 64, and 92. Pain and function scores in the affected shoulder were assessed using the ASES Subject Self-Evaluation form [7] (Table 2). The VAS pain

Table 3. Results at 30 days after $\mathrm{CCH}$ injection

\begin{tabular}{|c|c|c|c|c|}
\hline \multirow[b]{2}{*}{ Clinical characteristics } & \multirow[b]{2}{*}{ Placebo $(\mathrm{n}=15)$} & \multicolumn{3}{|l|}{$\mathrm{CCH}$ injections } \\
\hline & & $0.145 \mathrm{mg}(\mathrm{n}=16)$ & $0.29 \mathrm{mg}(\mathrm{n}=15)$ & $0.58 \mathrm{mg}(\mathrm{n}=14)$ \\
\hline Active elevation & $125^{\circ}$ & $132^{\circ}$ & $130^{\circ}$ & $140^{\circ \S}$ \\
\hline Active external rotation & $42^{\circ}$ & $42^{\circ}$ & $53^{\circ \mathrm{c}}$ & $49^{\circ}$ \\
\hline \multirow[t]{2}{*}{ Active internal rotation (spine level behind back) } & $8^{\circ}$ & $8^{\circ}$ & $8^{\circ}$ & $8^{\circ}$ \\
\hline & (L2) & (L2) & (L2) & (L2) \\
\hline Passive elevation & $146^{\circ}$ & $146^{\circ}$ & $149^{\circ}$ & $146^{\circ}$ \\
\hline Passive external rotation & $45^{\circ}$ & $49^{\circ}$ & $56^{\circ}$ & $60^{\circ \S}$ \\
\hline Passive external rotation at $90^{\circ}$ & $84^{\circ}$ & $82^{\circ}$ & $89^{\circ}$ & $88^{\circ}$ \\
\hline Function score* & 43 & 44 & 49 & $50^{\S}$ \\
\hline Pain score ${ }^{\dagger}$ & 3 & 3 & 4 & 3 \\
\hline
\end{tabular}

$\mathrm{CCH}=$ collagenase clostridium histolyticum; $* 60$ is optimal; ${ }^{\dagger} 5$ is no pain; ${ }^{\S} \mathrm{p}<0.05$ versus placebo (analysis of covariance). 
score ranged from zero (no pain at all) to 10 (pain as bad as it can be). Each item on the functional scale was answered on a four-point scale of 0 (unable to do), 1 (very difficult), 2 (somewhat difficult), and 3 (normal).

\section{Statistical Analysis, Study Size}

\section{Study 1. One CCH Injection}

An analysis of covariance model was used for the doubleblind phase of the study to adjust the four doses to the same treated-shoulder baseline for the specific subset of eight outcome measures: active elevation, active external rotation, active internal rotation, passive elevation, passive external rotation, passive external rotation at $90^{\circ}$, function score, and pain score. The analysis of covariance model consisted of terms for the covariate (treated shoulder at baseline) and the treatment group. Separate analyses were completed for each of eight outcome measures. Measurements were obtained at baseline for affected shoulders and unaffected contralateral shoulders. For the open-label phase of the study, means and SDs were calculated for the eight outcome measures on Days 1, 2, 4, 7, 14, and 30 after each injection. After Day 30, outcome measures were evaluated at 2, 3, 4, 6, and 12 months postinjection.

\section{Study 2. Volunteers Without Adhesive Capsulitis}

No Statistics (observational).

\section{Study 3. CCH Dose Range-volume versus Exercise-only Controls}

Data were summarized using descriptive statistics. A oneway ANOVA was used to compare primary and secondary efficacy variables according to cohorts, with two-sided $\mathrm{p}$ values. Patient satisfaction with treatment and investigator assessment of improvement with treatment were analyzed via the Wilcoxon rank-sum test with two-sided $p$ values. Self-reported compliance with the home exercise regimen was defined as compliance of "at least $75 \%$ of the time" reported at more than $50 \%$ of the times assessed.

\section{Results}

Study 1. One CCH Injection

When stratified by treatment group after one $\mathrm{CCH}$ injection, no improvements in individual parameters met the criterion of the minimally clinically important difference (Table 3). Therefore, one $\mathrm{CCH}$ injection did not provide clinically important improvements in ROM, function, or pain scores. As an example of a primary efficacy variable, at 30 days for active forward elevation (flexion) the means in degrees for placebo, $0.145 \mathrm{mg} \mathrm{CCH}, 0.29 \mathrm{mg} \mathrm{CCH}$, and $0.58 \mathrm{mg} \mathrm{CCH}$ were $125^{\circ}, 132^{\circ}, 130^{\circ}$, and $140^{\circ}(\mathrm{p}<0.01$ for $0.58 \mathrm{mg}$ versus placebo), respectively. Regarding adverse events, $100 \%$ of patients who received $\mathrm{CCH}$ injections experienced injection-site tenderness, which resolved in a mean of $5 \pm 4$ days, and biceps ecchymosis, which resolved in a mean of $9 \pm 5$ days. Six of 60 patients $(10 \%)$ had mild injection-area edema, which resolved in a mean of $1 \pm 0.7$ days. Forty-six of the 60 patients $(76 \%)$ entered the open-label phase as they did not reach treatment thresholds with either one placebo or $\mathrm{CCH}$ (various doses) injection. Forty-five (98\%) of these patients reached treatment thresholds with one or two additional $0.58-\mathrm{mg} /$ 0.5-mL CCH injections, 5 to 6 weeks apart. The majority $(\mathrm{n}=24)$ responded to one or two additional injections $(n=20)$. Adverse events were as described above. There were no adverse events or recurrence of adhesive capsulitis at 1 to 5 years of long-term followup among the patients who were accounted for.

\section{Study 2. Volunteers Without Adhesive Capsulitis}

In all 10 volunteers the injected saline fluid remained anterior to the shoulder capsule (confirmed by the posterior imaging) and extraarticular. Specifically, 10 volunteers showed a bolus (pocket) of saline fluid deep to the subscapularis tendon. No saline fluid was visible in the glenohumeral joint space by anterior or posterior ultrasound imaging. The saline fluid dissipated in all volunteers in a mean 4 minutes, 55 seconds ( \pm 55 seconds). No volunteer experienced any serious adverse effects after injection.

Study 3. CCH Dose Range-volume versus Exerciseonly Controls

Mean active forward flexion at baseline was $102^{\circ}$ to $115^{\circ}$ across cohorts and increased by Day 92 in all cohorts (Fig. 3). An improvement from baseline was observed in the primary endpoint of active forward flexion for patients in Cohort 2 compared with exercise-only Cohort 5 (38 versus $\left.12^{\circ} ; \mathrm{p}=0.039\right)$. This also was observed for active forward flexion in Cohort 3 compared with exercise-only Cohort $5\left(43^{\circ}\right.$ versus $\left.12^{\circ} ; \mathrm{p}=0.013\right)$. With the numbers available, no differences were observed for active abduction, active external rotation, and active internal rotation. 


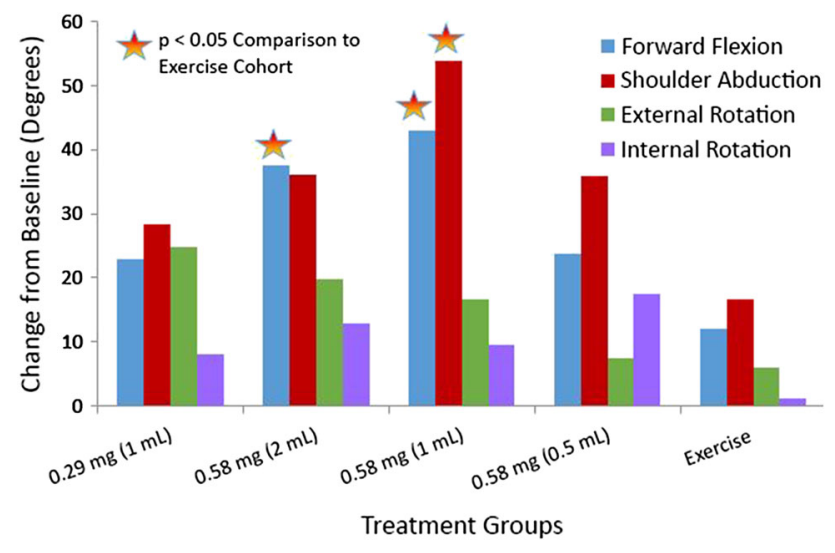

Fig. 3 The mean change in degrees for active ROM from baseline to Day 92 in Study 3 is shown. The $X$ axis shows the treatment groups consisting of the $\mathrm{CCH}$ dose cohorts and the exercise group. The $\mathrm{Y}$ axis shows degrees. $\mathrm{CCH}=$ collagenase clostridium histolyticum.

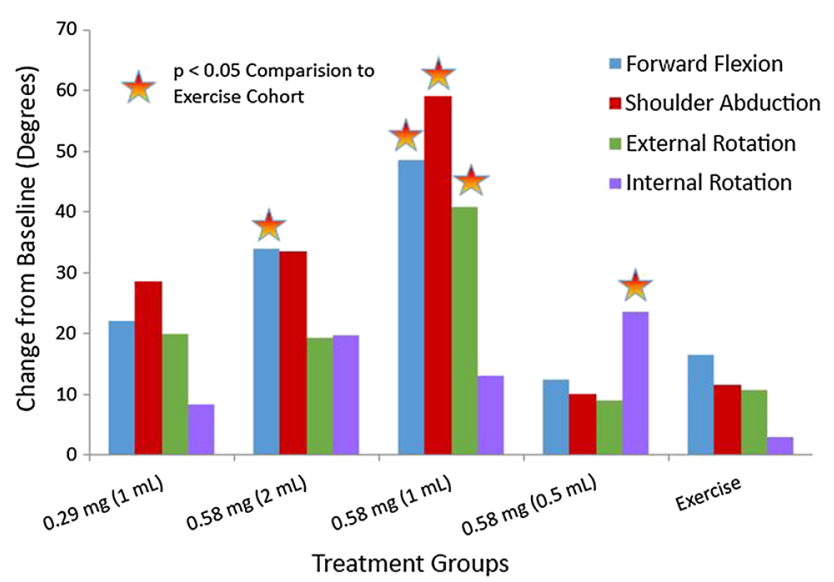

Fig. 4 The mean change in degrees for passive ROM from baseline to Day 92 in Study 3 is shown. The $\mathrm{X}$ axis shows the treatment groups consisting of the $\mathrm{CCH}$ dose cohorts and the exercise group, and the $\mathrm{Y}$ axis shows degrees. $\mathrm{CCH}=$ collagenase clostridium histolyticum.

Improvement was seen in active abduction $\left(54^{\circ}\right.$ versus $17^{\circ}$; $\mathrm{p}=0.005)$ when Cohort 3 was compared with exerciseonly Cohort 5.

Passive forward flexion and abduction and passive external and internal rotation also improved in the $\mathrm{CCH}$ treatment cohorts compared with the exercise-only cohort (Fig. 4). Greater improvements for passive forward flexion $\left(39^{\circ}\right.$ versus $\left.12^{\circ} ; \mathrm{p}=0.003\right)$ were observed for Cohort 2 compared with Cohort 5. Greater improvements for passive forward flexion $\left(46^{\circ}\right.$ versus $\left.12^{\circ} ; \mathrm{p}<0.001\right)$, passive abduction $\left(56^{\circ}\right.$ versus $\left.24^{\circ} ; \mathrm{p}=0.028\right)$, and passive external rotation $\left(41^{\circ}\right.$ versus $\left.11^{\circ} ; \mathrm{p}=0.010\right)$ were observed for Cohort 3 compared with Cohort 5 . In addition, greater improvements for passive internal rotation $\left(24^{\circ}\right.$ versus $3^{\circ}$; $\mathrm{p}=0.024)$ were observed in Cohort 4 compared with Cohort 5.

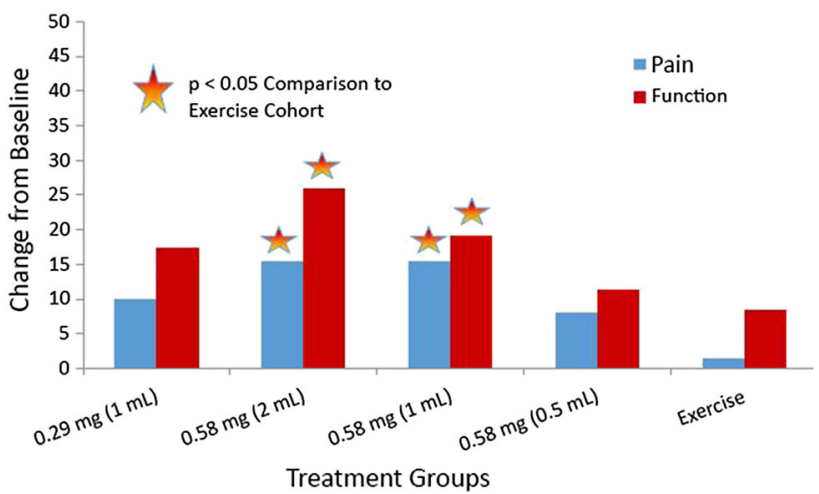

Fig. 5 The mean differences in VAS pain and ASES function scores from baseline to Day 92 in Study 3 are shown. The $\mathrm{X}$ axis shows the treatment groups consisting of the $\mathrm{CCH}$ dose cohorts and the exercise group. The $\mathrm{Y}$ axis shows the score values. ASES $=$ American Shoulder and Elbow Surgeons; $\mathrm{CCH}=$ collagenase clostridium histolyticum.

When Cohorts 2 and 3 were compared with exerciseonly Cohort 5, improvement was observed in VAS pain and ASES function scores (Fig. 5).

Patients who received $\mathrm{CCH}$ had adverse events (100\%) across $\mathrm{CCH}$ dose cohorts, compared with 10 patients (20\%) of the exercise-only cohort. Most treatment-related adverse events were confined to the local injection site and included injection-site pain (40-60\% of patients across $\mathrm{CCH}$ dose levels), bruising (30\%-60\%), swelling (20-50\%), and ecchymosis $(0-20 \%)$. The only other treatment-related adverse event to occur in greater than $20 \%$ of patients at any dose level was musculoskeletal pain (10-40\%). All adverse events resolved in 14 days or less without intervention. No treatment-related adverse events were considered severe or serious. None caused discontinuation from treatment or from the study. There were no adverse events in the exercise group.

MRI evaluations indicated no rotator cuff injuries or other clinically important safety findings from baseline to Day 92 in any patient. There were no clinically important changes in vital signs or clinical laboratory parameters.

\section{Discussion}

Conservative and even surgical treatments for adhesive capsulitis often result in prolonged limitation of ROM and pain for patients. Conservative treatments consisting of physical therapy, with or without steroid injection for adhesive capsulitis, take an extended toll on patients in terms of pain, attending therapy visits and/or doing therapy at home, loss of time from work, and activities of daily living. If a conservative therapy such as a $\mathrm{CCH}$ injection is developed, with FDA approval, it may have merit in 
restoring shoulder ROM and reducing pain in a shorter time.

Our study has some limitations, including small study numbers and the variability of diagnosing adhesive capsulitis by clinical examination only in Study 1. Diagnosis of adhesive capsulitis generally is one of exclusion of other shoulder disorders. MRI is useful in excluding other disorders although it cannot define a diagnosis of adhesive capsulitis. Therefore, in Study 1, some patients who had other shoulder disorders might have been enrolled. We relied on a diagnosis of adhesive capsulitis in all the $\mathrm{CCH}$ studies, on active and passive ROM restrictions, and especially in active and passive internal and external rotation. Another limitation of Study 1 was the short, 30day followup after one $\mathrm{CCH}$ injection. Patients then were eligible for an open-label extension phase using $0.58 \mathrm{mg}$ $\mathrm{CCH}$, where most received one or two additional $0.58-\mathrm{mg}$ $\mathrm{CCH}$ injections. Study 1, with the open-label extension phase allowed for assessments of $\mathrm{CCH}$ safety. Although we observed no serious adverse events, especially rotator cuff tears, after $\mathrm{CCH}$ injection(s), the possibility exists that these might occur. There was considerable patient dropout during the 12- to 60-month longer-term followup. Limitations also include the lack of a placebo group for Study 3; however, this study was designed as a comparative study versus a conservative standard of treatment. As an example, in Study 3, the majority of patients $(64 \%)$ had prior conservative adhesive capsulitis treatment that failed, consisting of steroid injection only (seven patients), steroid injection plus supervised physical therapy (15 patients), and supervised physical therapy only (10 patients).

In Study 1, our data indicated with the dose ranges studied, one injection of $\mathrm{CCH}$ was not sufficient to restore ROM and function and provide pain relief. The open-label extension option for this study confirmed that subsequent 0.58-mg CCH injections had merit and were well tolerated.

In Study 2 we confirmed that, using ultrasound guidance in the injection method, the injections to the anterior shoulder capsule were extraarticular. This is extremely important because the type II cartilage of the glenohumeral joint and other normal collagen-containing structures are potentially at risk for degradation by collagenase.

In Study 3, our data indicated that extraarticular ultrasound-guided injection(s) of $\mathrm{CCH}$ combined with standardized home shoulder exercises increased active forward flexion, our primary measure of efficacy. Improvements compared with exercise only were observed at the $0.58-\mathrm{mg}$ dose level of $\mathrm{CCH}$ when injected in volumes of $1 \mathrm{~mL}$ or $2 \mathrm{~mL}$. The $0.58-\mathrm{mg}$ dose level of $\mathrm{CCH}$ when injected in these volumes also improved additional secondary efficacy measures of ROM. Likewise, function was increased and pain decreased. Most patients in Study 3 received three injections of $\mathrm{CCH}$. This is consistent with studies of $\mathrm{CCH}$ for Dupuytren's contracture [1, 5, 10] and in clinical trials of $\mathrm{CCH}$ for Peyronie's disease using multiple injections [3]. Compared with other clinical studies of conservative treatment of adhesive capsulitis [2, $6,8,9,11], \mathrm{CCH}$ injections in Study 3 allowed patients to achieve improved ROM, functional status, and pain relief in a shorter time. This would be a distinct advantage to patients if $\mathrm{CCH}$ injections are approved by the FDA.

The theoretical mechanism of action of $\mathrm{CCH}$ could be loosening of the fibrosis involving the glenohumeral capsule and coracohumeral ligaments of the rotator interval by selective and targeted lysis of collagen fibers. We saw no evidence of clinically important damage to the rotator cuff or articular cartilage. MRI performed at baseline and at the end of Study 3 documented this. Other collagen-containing structures around the shoulder may be at risk.

We suggest that $\mathrm{CCH}$ injection(s) may have merit for patients with adhesive capsulitis in restoring shoulder ROM, increasing function, and reducing pain. Additional FDA-regulated clinical trials are planned which will compare collagenase injection(s) with a placebo in larger numbers of patients in randomized and blinded studies.

Acknowledgments We thank Kathleen Euler (studies coordinator, Department of Orthopaedics, Stony Brook University Medical Center, Stony Brook, NY) for help with all studies, and James Paci MD (Department of Orthopaedics, Stony Brook University Medical Center) for help with patient ROM assessments.

\section{References}

1. Badalamente MA, Hurst LC. Efficacy and safety of injectable mixed collagenase subtypes in the treatment of Dupuytren's contracture. J Hand Surg Am. 2007;32:767-774.

2. Blanchard V, Barr S, Cerisola FL. The effectiveness of corticosteroid injections compared with physiotherapeutic interventions for adhesive capsulitis: a systematic review. Physiotherapy. 2010; 96:95-107.

3. Gelbard M, Goldstein I, Hellstrom WJ, McMahon CG, Smith T, Tursi J, Jones N, Kaufman GJ, Carson CC 3rd. Clinical efficacy, safety and tolerability of collagenase clostridium histolyticum for the treatment of Peyronie's disease in 2 large double-blind, randomized, placebo controlled phase 3 studies. $J$ Urol. 2013;190:199-207.

4. Hawkins RJ, Bokor DJ. Clinical evaluation of shoulder problems. In: Matsen FA, ed. The Shoulder. Philadelphia, PA: W.B. Saunders; 1998:164-197.

5. Hurst LC, Badalamente MA, Hentz VR, Hotchkiss RN, Kaplan FT, Meals RA, Smith TM, Rodzvilla J; CORD I Study Group. Injectable collagenase clostridium histolyticum for Dupuytren's contracture. N Engl J Med. 2009;361:968-979.

6. Kelley MJ, McClure PW, Leggin BG. Frozen shoulder: evidence and a proposed model guiding rehabilitation. J Orthop Sports Phys Ther. 2009;39:135-148.

7. Michener LA, McClure PW, Sennett BJ. American Shoulder and Elbow Surgeons Standardized Shoulder Assessment Form, patient self-report section: reliability, validity, and responsiveness. J Shoulder Elbow Surg. 2002;11:587-594. 
8. Neviaser AS, Hannafin JA. Adhesive capsulitis: a review of current treatment. Am J Sports Med. 2010;38:2346-2356.

9. Neviaser AS, Neviaser RJ. Adhesive capsulitis of the shoulder. $J$ Am Acad Orthop Surg. 2011;19;536-542.

10. Peimer CA, Blazar P, Coleman S, Kaplan TD, Smith T, Tursi JP, Cohen B, Kaufman GJ, Lindau T. Dupuytren's contracture recurrence following treatment with collagenase clostridium histolyticum (CORDLESS): 3-year data. J Hand Surg Am. 2013;38:12-22.
11. Shah N, Lewis M. Shoulder adhesive capsulitis: systemic review of randomized trials using multiple corticosteroid injections. $\mathrm{Br} J$ Gen Practice 2007;57:662-667.

12. Xiaflex ${ }^{\circledR}$. Collagenase clostridium histolyticum. Auxilium Pharmaceuticals, Inc. Available at: http://www.endo.com/File\% 20Library/Products/Prescribing\%20Information/Xiaflex_prescribing information.html. Accessed March 8, 2016. 